REVIEW ARTICLE

F. Agosta

A. Chiò

M. Cosottini

N. De Stefano

A. Falini

M. Mascalchi

M.A. Rocca

V. Silani

G. Tedeschi

M. Filippi

\section{The Present and the Future of Neuroimaging in Amyotrophic Lateral Sclerosis}

SUMMARY: In patients with ALS, conventional MR imaging is frequently noninformative, and its use has been restricted to excluding other conditions that can mimic ALS. Conversely, the extensive application of modern MR imaging-based techniques to the study of ALS has undoubtedly improved our understanding of disease pathophysiology and is likely to have a role in the identification of potential biomarkers of disease progression. This review summarizes how new MR imaging technology is changing dramatically our understanding of the factors associated with ALS evolution and highlights the reasons why it should be used more extensively in studies of disease progression, including clinical trials.

\begin{abstract}
ABBREVIATIONS: ALS = amyotrophic lateral sclerosis; ALSFRS = ALS Functional Rating Scale; Cho $=$ choline; $\mathrm{Cr}=$ creatine; $\mathrm{CST}=$ corticospinal tract; $\mathrm{DTI}=$ diffusion tensor imaging; $\mathrm{FA}=$ fractional anisotropy; FLAIR = fluid-attenuated inversion recovery; $\mathrm{FMRI}=$ functional $M R$ imaging; $F T D=$ frontotemporal dementia; FUS/TLS = fused in sarcoma/translocated in liposarcoma gene; GM = gray matter; ${ }^{1} \mathrm{H}-\mathrm{MR}$ spectroscopy = proton MR spectroscopy; $\mathrm{L}=$ left; $\mathrm{LMN}=$ lower motor neuron; $M D=$ mean diffusivity; mlns = myo-inositol; $M T=$ magnetization transfer; $M T R=M T$ ratio; $\mathrm{NAA}=\mathrm{N}$-acetylaspartate; $\mathrm{ns}=$ not significant; $\mathrm{PD}=$ proton density; $\mathrm{R}=$ right; $\mathrm{SOD} 1=$ superoxide dismutase $1 ; \mathrm{SPM}=$ statistical parametric mapping; TDP-43 = TAR DNA-binding protein gene; $\mathrm{UMN}=$ upper motor neuron; $\mathrm{VBM}=$ voxel-based morphometry; $\mathrm{WM}=$ white matter
\end{abstract}

A LS, also known as motor neuron disease, is a neurodegenerative disorder characterized by a progressive muscular paralysis reflecting degeneration of motor neurons in the primary motor cortex, brain stem, and spinal cord. The phenotypic expression of ALS is highly heterogeneous and determined by 4 elements: 1) body region of onset, 2) relative mix of UMN and LMN involvement, 3) rate of progression, and 4 ) cognitive impairment. ${ }^{1}$ In approximately $5 \%-10 \%$ of patients, the disease is inherited; $20 \%$ of these individuals have a mutation of the SOD1 gene; approximately $2 \%-5 \%$, of the TARDBP (TDP-43) gene; and 2\%-4\%, of the FUS/TLS gene. ${ }^{2}$ Most patients with ALS, however, have no obvious family history and have sporadic ALS. ${ }^{2}$ To date, the only specific marker of sporadic ALS is the presence of inclusions staining positively for ubiquitin and TDP-43 in degenerating motor neurons. ${ }^{3}$

Despite technical advances in medicine in the last century, the diagnosis of sporadic ALS relies on the interpretation of clinical symptoms and signs (ie, signs suggestive of combined UMN and LMN degeneration, together with disease progression compatible with a neurodegenerative disorder). Paraclinical and laboratory tests are used only to ex-

From the Neuroimaging Research Unit (F.A., M.A.R., M.F.), Institute of Experimental Neurology, and Neuroradiology Unit (A.F.), Division of Neuroscience, Scientific Institute and University Hospital San Raffaele, Milan, Italy; Department of Neuroscience (A.C.), University of Torino, Turin, Italy; Neuroradiology Unit (M.C.), Department of Neuroscience, University of Pisa, Pisa, Italy; Department of Neurological and Behavioral Sciences (N.D.S.), University of Siena, Siena, Italy; Radiodiagnostic Section (M.M.), Department of Clinical Physiopathology, University of Florence, Florence, Italy; Department of Neurology and Laboratory of Neuroscience (V.S.), "Dino Ferrari” Center, Università degli Studi di MilanoIRCCS Istituto Auxologico Italiano, Milan, Italy; Department of Neurological Sciences (G.T.), II University of Naples, Naples, Italy; and Institute Hermitage Capodimonte (G.T.), Naples, Italy.

Please address correspondence to Massimo Filippi, MD, Neuroimaging Research Unit, Institute of Experimental Neurology, Division of Neuroscience, Scientific Institute and University Hospital San Raffaele, Via Olgettina, 60, 20132 Milan, Italy; e-mail: m.filippi@hsr.it

Indicates open access to non-subscribers at www.ajnr.org

DOI 10.3174/ajnr.A2043 clude "ALS-mimic" syndromes. ${ }^{4,5}$ In ALS, the absence of a disease marker for UMN and LMN involvement has 2 main negative consequences. First, the delay from onset of the disease to diagnosis of ALS can vary between 13 and 18 months ${ }^{6,7}$; and the diagnostic delay may even be greater in patients who present with isolated LMN signs. ${ }^{8}$ Such a delay precludes early initiation of neuroprotective treatments. ${ }^{9}$ Second, the phenotypic heterogeneity of the disease is an important confounding factor in clinical trials because it results in a limited inclusion of patients (which requires the fulfillment of the revised El Escorial criteria $^{10}$ ) and in a highly variable treatment response.

Several neuroimaging modalities have been used with varying success either to aid the clinical process of establishing a diagnosis of sporadic ALS or to monitor disease progression. ${ }^{11}$ Conventional MR imaging of the brain and spinal cord has an important role in the differential diagnosis. ${ }^{12}{ }^{1} \mathrm{H}-\mathrm{MR}$ spectroscopy and DTI hold promise for detecting and quantifying subclinical UMN damage. ${ }^{11}$ The use of fMRI has provided evidence for cortical reorganization in patients with ALS and has contributed to achieving a more complete picture of the ALS-related extramotor involvement. ${ }^{13}$ This review summarizes the main results obtained from the application of conventional and nonconventional MR imaging in patients with ALS and highlights many areas for which more research is warranted.

\section{Conventional MR Imaging Findings}

Routine anatomic imaging of the brain and/or the spinal cord is helpful in ruling out diseases that mimic ALS with varying degrees of UMN and LMN signs. ${ }^{5}$ The revised criteria of the World Federation of Neurology Research Group on Motor Neuron Diseases ${ }^{10}$ state that conventional MR imaging studies are not required in those cases that have a clinically definite disease with a bulbar or a pseudobulbar onset. On the other hand, in patients with clinically probable or possible ALS, MR imaging can be useful in excluding several 

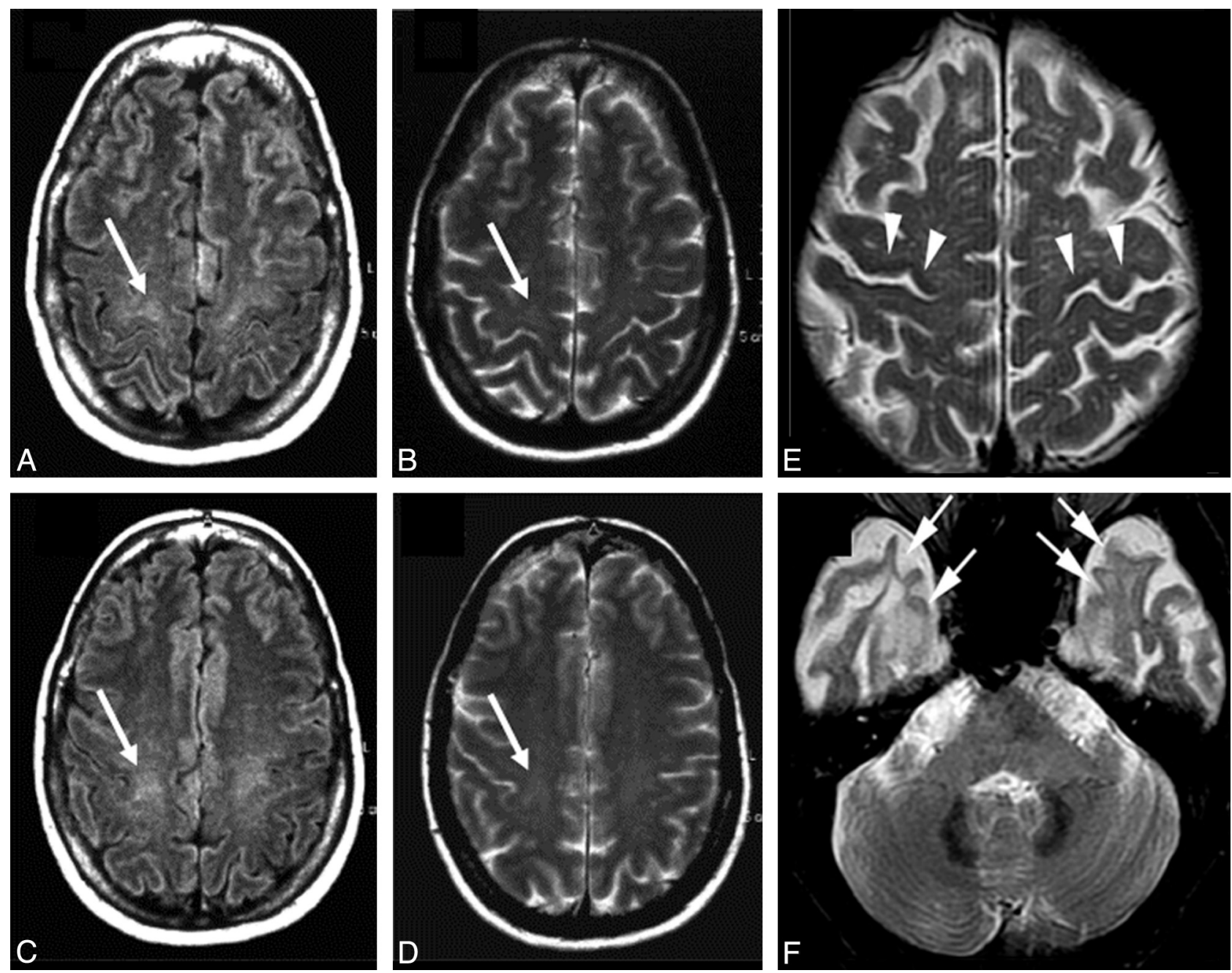

Fig 1. Conventional MR imaging findings in patients with ALS. $A-D$, Hyperintensity at the subcortical precentral gyrus $(A$ and $B)$ and the centrum semiovale $(C$ and $D)$ on FLAlR (arrows, $A$ and $C$ ) and T2-weighted (arrows, $B$ and $D$ ) images of a 66-year-old patient with ALS. Modified with permission from Hecht et al. ${ }^{17} E$ and $F$, T2-weighted images obtained from a 58-year-old patient with ALS with dementia show hypointensity along the precentral cortices (arrowheads, E) and symmetric hyperintensity in the anterior temporal subcortical WM (arrows, F). A and D reprinted with permission from the Journal of the Neurological Sciences (2001;186:37-44). Copyright 2001, Elsevier Ltd.

ALS-mimic syndromes, including cerebral lesions (eg, multiple sclerosis and cerebrovascular disease), skull base lesions, cervical spondylotic myelopathy, other myelopathy (eg, foramen magnum lesions, intrinsic and extrinsic tumors, and syringomyelia), conus lesions, and thoracolumbar sacral radiculopathy. ${ }^{12}$

In patients with ALS, signal-intensity changes on conventional MR imaging (ie, T2-weighted, PD-weighted, and FLAIR sequences) can be observed along the CST. ${ }^{14-23}$ Typically, CST changes, which are best followed on coronal scans, appear as areas of bilateral increased signal intensity from the centrum semiovale to the brain stem. However, the frequency of CST hyperintensities in patients with ALS varies significantly in the different studies (ranging from $15 \%$ to $76 \%$ ), and it is not clear yet which may be the most sensitive MR image to detect them (Fig $1 A-D$ ). The combined application of T2-weighted, PDweighted, and FLAIR images has recently reached a sensitivity of approximately $62 \% .{ }^{24}$ In addition, CST signal-intensity changes do not correlate with clinical scores. ${ }^{17}$ More remarkably, CST hyperintensities have also been described in healthy subjects, ${ }^{20}$ and in patients with other conditions, such as
Krabbe disease, ${ }^{25} \mathrm{X}$-linked Charcot-Marie Tooth neuropathies, ${ }^{26}$ and adrenomyeloneuropathy. ${ }^{27}$

In patients with ALS, a characteristic low signal intensity (hypointense rim) of the precentral cortex on T2-weighted images can be observed (Fig 1E). ${ }^{16,18,19,21,22,28}$ However, such a change is neither sensitive nor specific to the pathology of ALS.

Symmetric T2 signal-intensity changes in the anterior temporal subcortical WM have been described in patients with ALS and dementia (Fig $1 F){ }^{29,30}$ Pathologically, such hyperintensities correspond to loss of myelin, ${ }^{30} \mathrm{WM}$ degeneration, ${ }^{29}$ and gliosis. ${ }^{29}$

Significant lower whole brain volume has been found in patients with ALS relative to healthy controls. ${ }^{31-33}$ Global brain atrophy is, however, relatively mild.

Consistent with pathologic data, T2 and T1 hyperintensities of the anterolateral columns of the cervical cord have been observed in patients with ALS. ${ }^{21,22,34}$ Such hyperintensities are likely to have higher specificity than signal-intensity changes on brain MR images. ${ }^{21,34} \mathrm{~T} 1$ hyperintensity of the anterolateral cervical cord has been associated with a younger age and a rapid disease progression. ${ }^{22}$ 


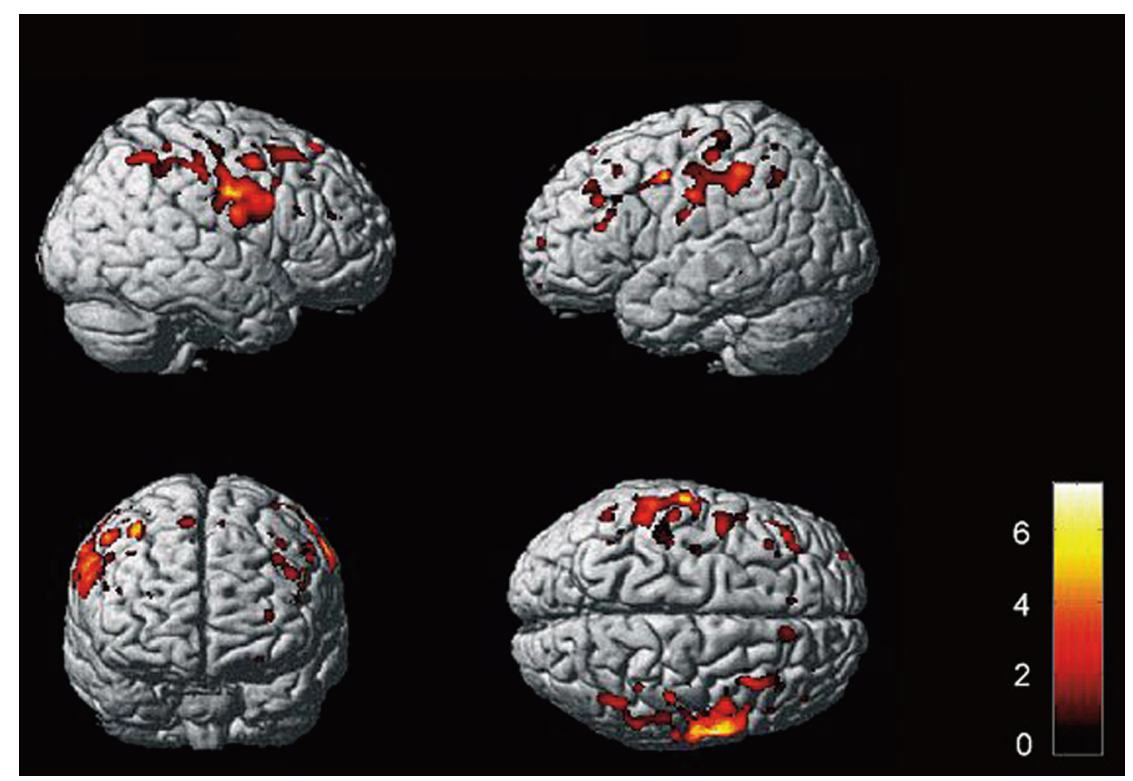

Fig 2. Regional GM atrophy in patients with ALS compared with controls. A group comparison of 17 patients with ALS versus 17 healthy controls ${ }^{37}$ shows regional GM atrophy in the precentral and postcentral gyri bilaterally, which extends from the primary motor cortex to the premotor, parietal, and frontal regions bilaterally (displayed at $P=.001$, uncorrected; extended threshold, 100 voxels). The color bar represents the $t$-score. The differences between groups are superimposed on a standard normalized T1-weighted image. Images are shown in neurologic convention.

\section{Nonconventional MR Imaging Assessment of the Motor System}

\section{Regional Atrophy}

Despite the consistent finding of cortical motor involvement in pathologic studies of ALS, ${ }^{35}$ in vivo MR imaging studies did not reach firm conclusions regarding the presence of motor/ premotor cortical atrophy because this was reported by some authors (Fig 2), ${ }^{31,36-42}$ but not by others. ${ }^{32,43-45}$

Using VBM to assess the regional distribution of WM loss, researchers have found atrophy of the CST in patients with ALS, ${ }^{31,44}$ which was more severe in patients with a bulbar onset. ${ }^{44}$ Patients with ALS showed atrophy of the cervical cord relative to controls, ${ }^{46}$ which significantly worsened during a 9-month follow-up. ${ }^{47}$

\section{${ }^{1} H$-MR Spectroscopy}

In patients with ALS, ${ }^{1} \mathrm{H}-\mathrm{MR}$ spectroscopy studies demonstrated a decrease in NAA concentrations ${ }^{48-50}$ and ratios of NAA over $\mathrm{Cr},{ }^{50-54} \mathrm{Cho},{ }^{51,55}$ and $\mathrm{Cr}+\mathrm{Cho}^{54}$ in the motor cortex. The physiologic role of NAA in the central nervous system remains unclear. However, because NAA appears to be present only in neurons of the adult human brain and in their processes, the content of NAA is used as a biochemical marker of neuronal integrity. ${ }^{56}$ These findings have been recently confirmed at 3T. ${ }^{57-59}$ However, the diagnostic value of ${ }^{1} \mathrm{H}-\mathrm{MR}$ spectroscopy is poor because of the considerable overlap of the metrics of patients with those of healthy controls.

Patients with ALS showed decreased NAA/Cr and NAA/ Cho ratios along the CST. Although the regional analysis of these data showed more pronounced ${ }^{1} \mathrm{H}-\mathrm{MR}$ spectroscopy changes in the precentral gyrus and corona radiata, ${ }^{60}$ NAA reduction in the brain stem has also been reported..$^{61}$

Cross-sectional studies have shown a moderate correlation between NAA concentration (and its ratios) in the motor cor- tex and clinical manifestations of ALS (ie, disease severity ${ }^{50,59,62,63}$ extent of UMN signs, ${ }^{64}$ and maximum finger tapping rate $\left.^{54}\right)$. Patients with bulbar onset have the most severe pattern of motor cortex ${ }^{1} \mathrm{H}-\mathrm{MR}$ spectroscopy changes compared with those with a limb onset.

In addition to neuronal damage, increased glial cell activity, reflected by raised levels of mIns, has been demonstrated in the motor cortex of patients with ALS. ${ }^{51,65}$ Increased mIns levels are associated with motor cortex hypointensity on T2weighted images. ${ }^{65}$ In a $3 \mathrm{~T}^{1} \mathrm{H}$-MR spectroscopy study of patients with ALS, ${ }^{57}$ the NAA/mIns ratio provided better sensitivity and specificity for detecting disease than the individual metabolites and was the strongest correlation with disease severity.

\section{DTI}

Using either region of interest-based approaches or tractography, DTI studies of patients with ALS reported consistently decreased FA and increased MD values along the CST (Fig 3). ${ }^{66-75}$ Loss of pyramidal motor neurons in the primary motor cortex and axonal degeneration of the CST, together with the proliferation of glial cells, extracellular matrix expansion, and intraneuron abnormalities, ${ }^{76}$ may contribute to the observed CST DTI changes. The most pronounced decreased FA and increased MD have been shown in the posterior limb of internal capsule. ${ }^{66-74}$

Decreased FA in patients with ALS was found to correlate with disease severity, ${ }^{59,66,67,71}$ rate of disease progression, ${ }^{74}$ and clinical ${ }^{67,69}$ and electrophysiologic ${ }^{70}$ measures of UMN involvement. Patients with a bulbar onset exhibited the most marked FA decrease. ${ }^{67}$ In 2 studies, increased MD of the CST has been associated with disease duration. ${ }^{66,67}$

Compared with controls, patients with ALS had significantly lower FA of the cervical cord, which was strongly associated with the ALSFRS scores (Fig 4$).{ }^{46}$ This finding indicates 

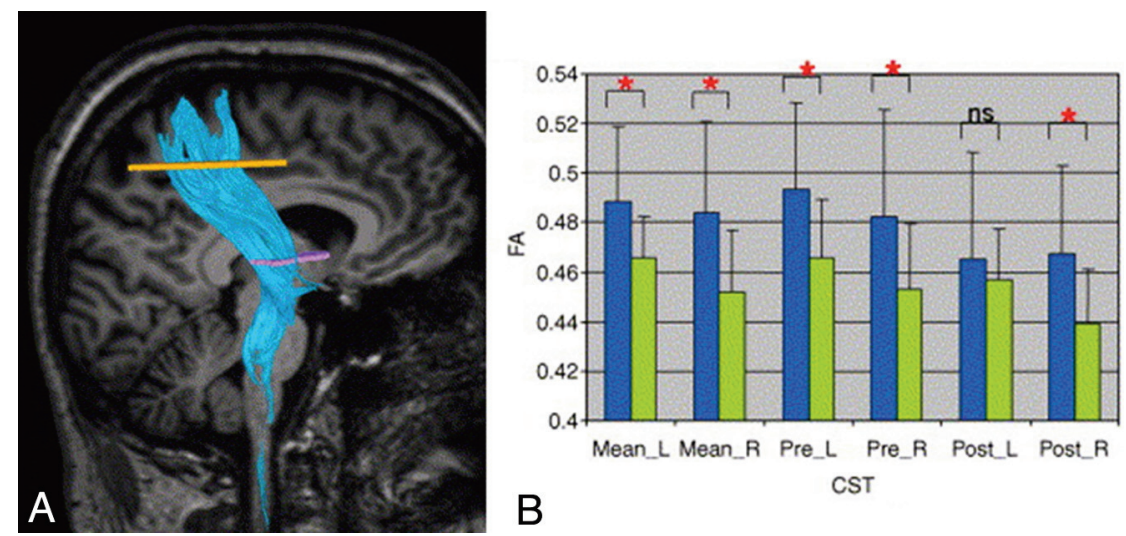

Fig 3. A, A representative example of DTI tractography of the CST. B, Graphs of mean FA in the right and left CSTs of 28 patients with ALS (green) and 26 healthy controls (blue). Data are presented for the entire CST (mean) and its pre- and postcentral portions. Mean values are shown with SDs (black lines). Significant differences in diffusion parameters between groups are indicated by a red asterisk. Reprinted with permission from Neuroimage (2007;34:486-99). Copyright 2007, Elsevier Ltd.
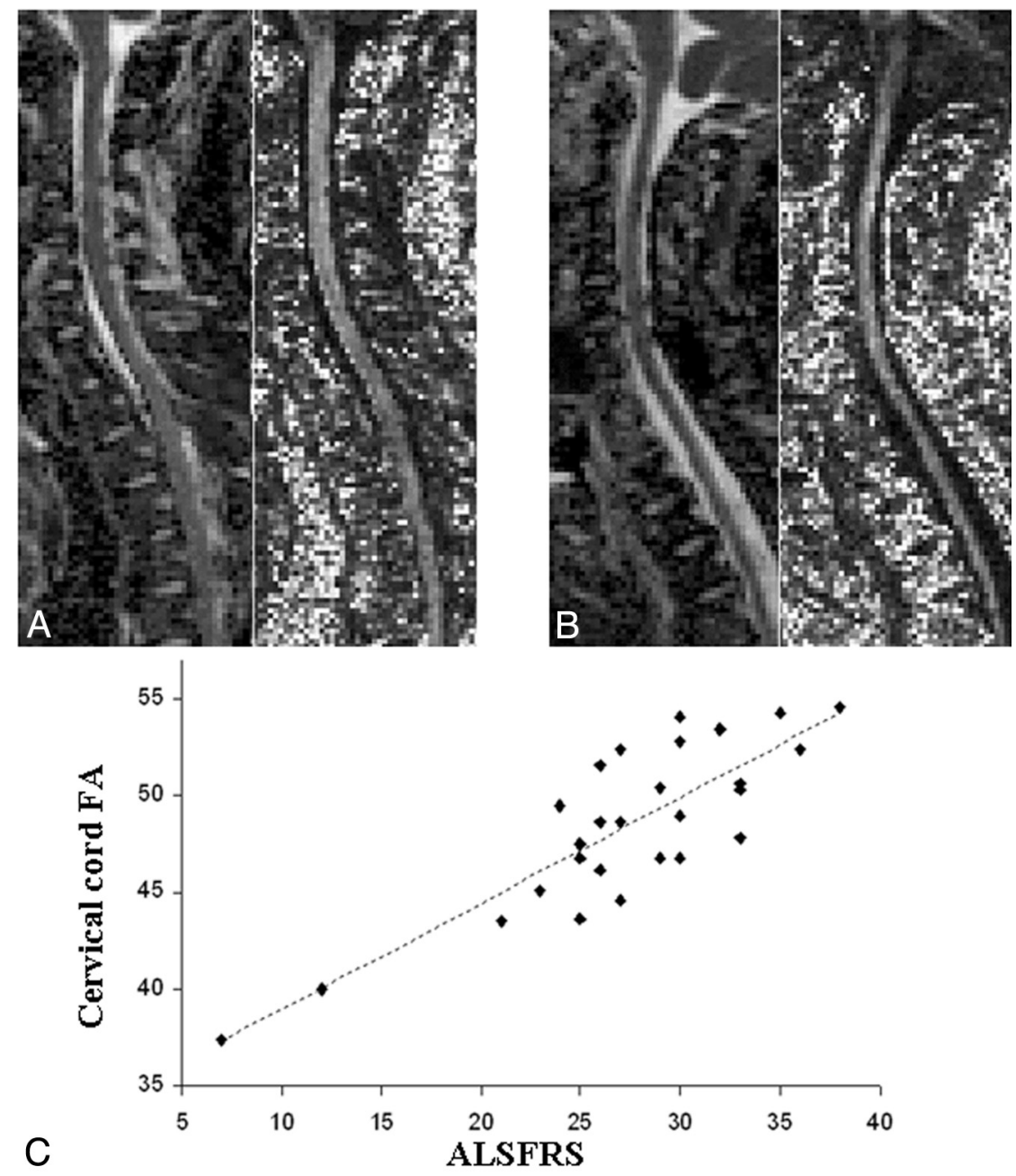

Fig 4. $A$ and $B$, Illustrative examples of MD (images on the left of each pair) and FA (images on the right of each pair) maps of the cervical cord obtained from a healthy volunteer ( $A$ and a patient with ALS $(B)$. C, Scatterplot of the correlation between the cervical cord FA and the ALSFRS in 28 patients with ALS. Reprinted with permission from the Journal of Neurology, Neurosurgery \& Psychiatry (2007;78:480-84). Copyright 2007, BMJ Publishing Group Ltd.

the presence of distortion of cord tissue geometry in ALS and agrees with pathologic data showing a pronounced cord degeneration and a reduction of the number of LMNs in the anterior horns of the cord GM in patients with ALS. ${ }^{77}$

\section{MT Imaging}

Using T1-weighted MT images, 1 study showed hyperintensities along the CST (consistent with CST degeneration) in $80 \%$ of patients with ALS. ${ }^{78}$ T1-weighted MT imaging provided 

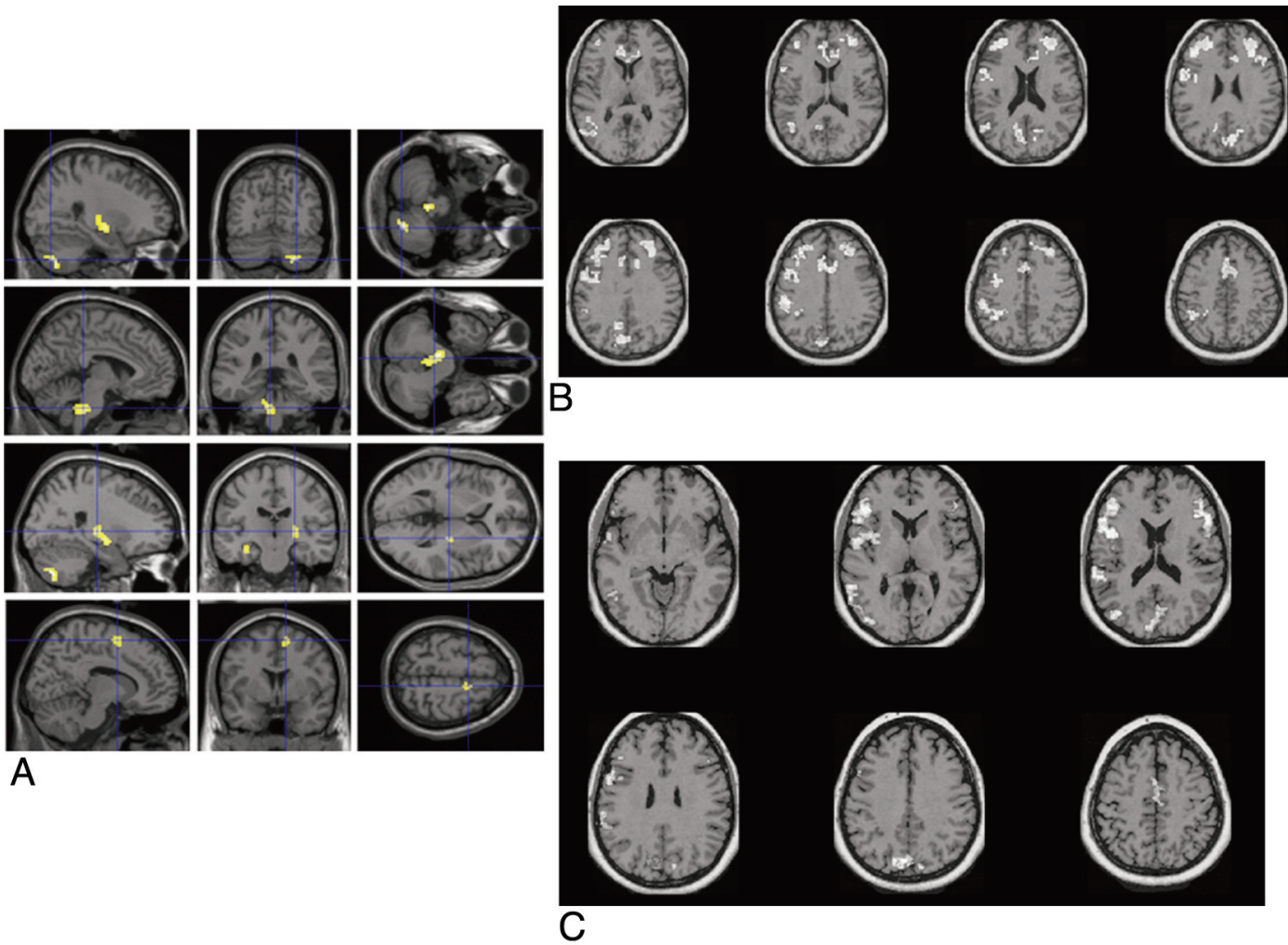

Fig 5. $A$, Brain regions showing a significant increased recruitment on $\mathrm{fMRI}$ scans from 10 patients with ALS relative to 10 healthy controls during right-handed movement $(P<.05$, smal volume correction). Modified with permission from Konrad et al. ${ }^{83} B$ and $C$, Areas of reduced fMRI activation in 28 nondemented patients with ALS relative to the control group during letter fluency $(B)$ and confrontation naming $(C)$ tasks. A reprinted with permission from Experimental Brain Research (2006;172:361-69). Copyright 2006, Springer Science + Business Media. $B$ and $C$ reprinted with permission from Brain (2004;127:1507-17). Copyright 2004, Oxford University Press.

better sensitivity and specificity for detecting disease than FLAIR images. $^{78}$

Two MT imaging studies showed a reduction of the MTR in the CST from $2.6 \%{ }^{79}$ to $20 \%^{80}$ in patients with ALS compared with controls. This finding was not confirmed by a recent study. ${ }^{24}$ MT contrast is the result of cross relaxation and chemical exchange between spins in the 2 pools of water protons: bound immobile protons associated with macromolecules (such as myelin) and free mobile protons associated with free water. MTR is a quantitative measurement of this phenomenon, and it is correlated to the amount of bound protons in the macromolecular matrix. Reduced MTR values have been associated with axonal degeneration and myelin breakdown. ${ }^{81}$ A possible explanation for the negative results in the study by Charil et $\mathrm{al}^{24}$ could be that gliosis secondary to axonal loss might have led to a pseudonormalization of the MTR.

\section{fMRI}

During motor tasks, fMRI demonstrated an increased activation of the contralateral sensorimotor cortex and the supplementary motor area (Fig 5A). ${ }^{82-85}$ Increased sensorimotor activation was also reported in the hemisphere ipsilateral to the movement. ${ }^{84}$ A spatial shift of recruitment to more anterior regions of the premotor cortex during upper limb movement was also observed in patients with ALS. ${ }^{82}$ In patients with ALS, increased activation of the inferior parietal cortex during a motor task has been reported. A relationship with task difficulty ${ }^{84}$ or a compensatory role of these motor-related $\operatorname{areas}^{82,85}$ has been suggested.
In ALS, motor execution was also associated with increased activations of areas involved in motor learning, such as the basal ganglia and the cerebellum (Fig 5A). ${ }^{83,86}$ It is suggested that an increase in movement-associated cortical activations beyond the primary motor cortex is associated with the degree of UMN involvement. ${ }^{86}$

\section{Nonconventional MR Imaging Assessment of the Extramotor Regions}

\section{Regional Atrophy}

The pattern of regional GM loss in patients with ALS extends beyond the motor cortex to the frontotemporal and parietal regions (Fig 2). ${ }^{31,32,36-38,40,41,44}$ The most severe atrophy of the frontal regions has been observed in patients with ALS and FTD. ${ }^{38}$ In addition, patients with ALS with even subtle cognitive and/or behavioral impairment (not meeting the criteria for a diagnosis of FTD ${ }^{87}$ ) demonstrated GM loss in the frontal, parietal, and limbic regions compared with those with no evidence of cognitive deficits. ${ }^{88}$ These findings are consistent with pathologic studies demonstrating ubiquitin-positive intraneuronal inclusions and neuronal loss extending beyond the motor system in patients with ALS with or without cognitive impairment. ${ }^{35}$

VBM studies in patients with ALS have provided evidence for WM atrophy in extramotor areas, which include the corpus callosum, the cerebellum, and the frontotemporal and occipital areas. ${ }^{31,33,45}$ In 1 study of cognitively impaired non- 

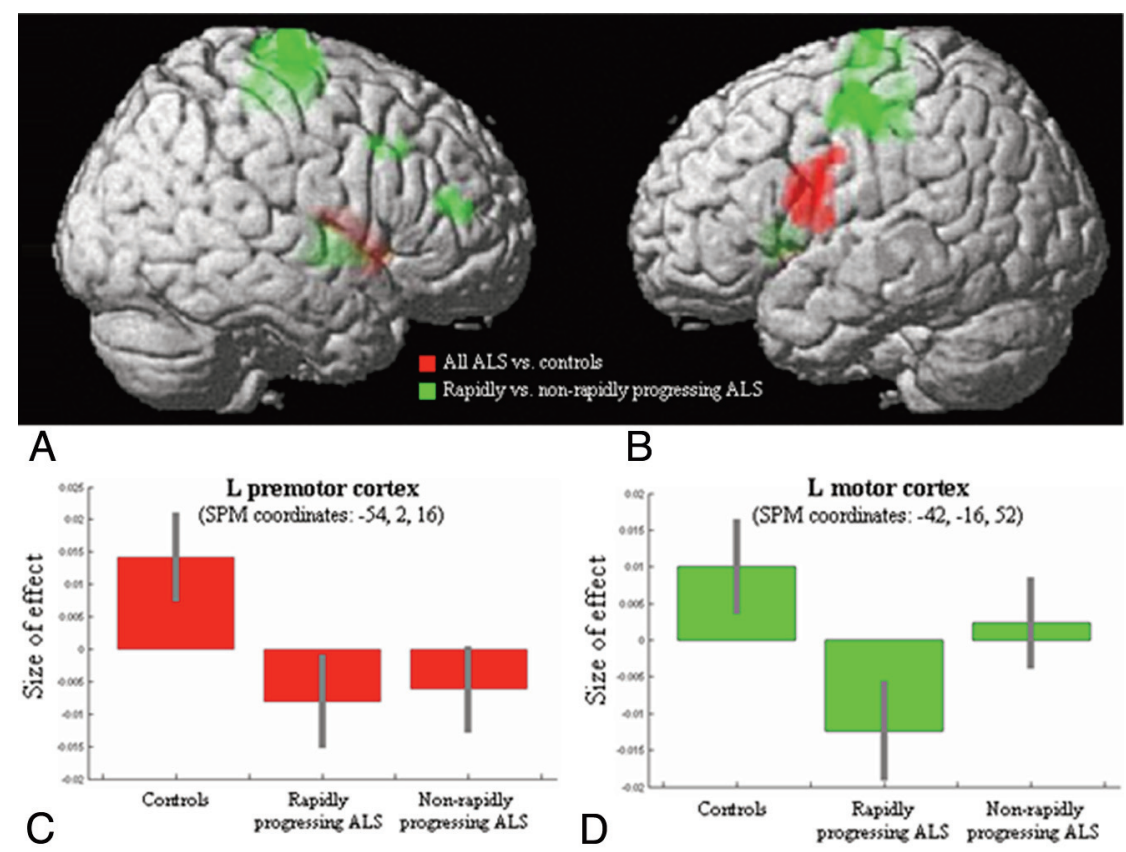

Fig 6. Regions showing progression of GM atrophy during a 9-month period ${ }^{90}$ in 16 patients with ALS compared with 10 controls (red) and regions showing additional GM loss in clinically rapidly progressing patients with ALS compared with both controls and nonrapidly progressing patients (green). $A$ and $B$, Results are superimposed on the $3 \mathrm{D}$ rendering of the Montreal Neurologic Institute standard brain and displayed at a threshold of $P<.05$, uncorrected. Color saturation depends on the position of the brain region in relation to the cortical surface in the rendered brain (ie, motor, premotor, and prefrontal regions are more saturated; basal ganglia are less saturated). $C$ and $D$, Plots showing the "size of effect" in the left premotor and motor cortices for each group of subjects (healthy controls, patients with rapidly progressing ALS, and those with nonrapidly progressing ALS). The "size of the effect" was obtained by plotting the estimates of the analysis of variance model parameters, which were of interest in the present analysis (3 subject groups). The bars represent the $90 \%$ confidence intervals. Reprinted with permission from Amyotrophic Lateral Sclerosis (2009;10:168-74). Copyright 2009, Taylor and Francis.

demented patients with ALS, a more severe frontotemporal WM loss was associated with deficits on verbal fluency. ${ }^{45}$

Significant longitudinal cortical atrophy during a period of $<1$ year has been described in motor and prefrontal areas in patients both with ${ }^{89}$ or without (Fig 6) ${ }^{90}$ clinical symptoms typical of FTD.

\section{${ }^{1}$ H-MR Spectroscopy}

In patients with ALS, a decrease of NAA levels is not restricted to the motor cortex and CST but also occurs in premotor regions, the primary sensory cortex, and extramotor frontal regions, with relative sparing of the parietal lobe. ${ }^{53,63}$ A correlation was found between the decrease in the NAA/Cr ratio in the frontal lobe and deficits of executive functions. ${ }^{63}$

\section{DTI}

In patients with ALS, FA decrease has been found in the corpus callosum $^{36,75,91-93}$ and in several WM regions in the fron$\operatorname{tal}^{75,91,93}$ and temporal lobes. ${ }^{75,93}$ The topographic pattern of MD changes in patients with ALS has been investigated by a few studies, which showed increased MD in the corpus callosum and in frontal and temporal WM regions compared with controls. ${ }^{36,93}$

\section{fMRI}

Significantly reduced activation of the middle and inferior frontal gyri, anterior cingulate cortex, and parietal and temporal lobes was found in nondemented patients with ALS relative to controls during a letter fluency fMRI task (Fig 5B). ${ }^{94} \mathrm{~A}$ confrontation naming fMRI task also revealed an impaired activation of a prefrontal region (including Broca area) and areas of the temporal, parietal, and occipital lobes (Fig 5C)..$^{94}$ This pattern of dysfunction correlated with cognitive deficits on both word fluency and confrontation naming. ${ }^{94}$

Functional cortical changes have been observed in patients with ALS without cognitive/behavioral impairment during processing of socioemotional stimuli (ie, pictures of persons in emotional situations). ${ }^{95}$ Compared with controls, patients with ALS showed an increased activation of the right supramarginal gyrus. ${ }^{95}$

\section{The Near Future of Neuroimaging of ALS}

\section{The Prognostic Value of MR Imaging}

In ALS, death due to respiratory failure follows on average 3-5 years after onset. However, the survival of individual patients with ALS is highly variable. A rapidly progressive course can occur, but approximately $10 \%$ of patients survive for a decade or more. ${ }^{96}$ Identifying predictors of progression and survival in ALS is important for both management of patients in clinical practice and design of new clinical trials. Older age at onset, bulbar site of onset, and a shorter time from symptom onset to diagnosis are the most consistently reported clinical predictors of shorter survival. A further contribution may come from MR imaging by the identification of disease changes earlier in the course of the disease. In one ${ }^{1} \mathrm{H}-\mathrm{MR}$ spectroscopy study, a reduced short-term survival has been associated with a lower NAA/Cho ratio. ${ }^{57}$

\section{MR Imaging to Monitor Disease Evolution}

Longitudinal neuroimaging studies are likely to contribute to the monitoring of ALS progression. To date, only a few studies 
have attempted to assess quantitatively the dynamics of nonconventional brain structural changes in patients with ALS, and they provided conflicting results. Some studies have demonstrated a significant decrease of the NAA level in the motor cortex $^{55,97-99}$ or the FA in the brain $\mathrm{WM}^{97}$ and cervical cord ${ }^{47}$ during $<1$ year. Changes of metabolite ratios and diffusivity abnormalities were significantly correlated with progression of disease severity ${ }^{55,97}$ A serial ${ }^{1} \mathrm{H}-\mathrm{MR}$ spectroscopy study indicated that a significant decrease of the NAA level also occurs in brain extramotor regions after 9 months. ${ }^{99}$ However, in 1 prospective study of 30 patients with ALS, no significant ${ }^{1} \mathrm{H}-\mathrm{MR}$ spectroscopy and DTI changes were observed. ${ }^{50}$ Similar negative findings have been found by others. ${ }^{47,100}$

The main limitation of longitudinal MR imaging studies in ALS is the small sample sizes used. In addition, patients recruited are typically advanced in their disease course, which may imply that structural changes due to ALS pathology had already occurred and stabilized at the time of MR imaging, with only small changes occurring thereafter. Finally, longitudinal MR imaging follow-up of patients with ALS is limited by the declining ability of the patients to tolerate the relatively long scanning time needed to obtain meaningful quantitative MR imaging data. Future investigations should involve larger groups of subjects, possibly early in the course of the disease.

Despite many clinical trials and various advances in the understanding of ALS, there has been little success in the search for disease-modifying or neuroprotective agents. At present, riluzole is the only approved drug that has been shown to have a modest effect on prolonging life expectancy in patients with ALS. ${ }^{96}$ The assessment of treatment effects on ${ }^{1} \mathrm{H}$-MR spectroscopy measures in ALS is only preliminary. A rapid increase of the $\mathrm{NAA} / \mathrm{Cr}$ ratio in the motor cortex in patients with ALS after only 1 day of riluzole treatment has been reported. ${ }^{101,102}$ Conversely, no effect on cortical metabolic function has been observed with gabapentin treatment ${ }^{103}$ or brain-derived neurotrophic factor. ${ }^{104}$ The reliability and reproducibility of measures of brain ${ }^{1} \mathrm{H}-\mathrm{MR}$ spectroscopy also warrant further investigation. In addition, the sensitivity and clinical relevance of ${ }^{1} \mathrm{H}-\mathrm{MR}$ spectroscopy scans in detecting longitudinal ALS-related changes need to be established before this technique is used for monitoring treatment efficacy in the context of multicenter clinical trials.

\section{Iron-Dependent MR Imaging}

Iron deposition is known to accumulate in the brain with normal aging, and recent studies have shown that iron is also involved in brain damage in many chronic neurologic disorders, such as Alzheimer disease, Parkinson disease, and multiple sclerosis. ${ }^{105}$ An abnormal iron deposition has been supposed to be the substrate of T2 hypointense areas seen in the cortex of patients with ALS. ${ }^{16,18,19,21,22,28} \mathrm{~T} 2$ and other sequences used at $\geq 3 \mathrm{~T}$, such as $\mathrm{T} 2^{\star}, \mathrm{T}^{\prime}{ }^{\prime}$, or $\mathrm{T} 2$-rho relaxometry; magnetic field correlation imaging and susceptibilityweighted imaging are likely to improve our ability to detect iron deposition. As a consequence, iron-dependent MR imaging may soon provide markers to monitor the progression of brain damage in many neurologic diseases, including ALS.

\section{$f M R I$}

The difficulty of controlling task performance in patients with ALS may be responsible for the variability of the results of fMRI studies. The development of fMRI paradigms unbiased by differences in task performance between patients with ALS and controls, making the assessment of more disabled patients feasible, should be considered. Motor imagery is known to involve similar neural networks such as motor execution, without being affected by confounding factors of effort and strain. During a motor imagery task, patients with ALS showed increased activation of the premotor and the primary motor cortex. ${ }^{106}$ After 6 months, these differences persisted with additional activations of the precentral gyrus and frontoparietal regions. ${ }^{106}$ In ALS, the resting-state fMRI studies demonstrated not only changes in the premotor cortex but also a reduced activation of the default mode network. ${ }^{107}$ Another aspect that should be considered is the combination of measures of functional connectivity with those of structural damage of specific WM tracts, which is likely to improve our understanding of the relationship between morphologic and functional abnormalities observed in this condition.

\section{Conclusions}

Findings compatible with ALS on conventional MR imaging are not consistently found and are nonspecific. By demonstrating evidence of occult UMN degeneration in vivo, ${ }^{1} \mathrm{H}-\mathrm{MR}$ spectroscopy and DTI may provide a faster and more definitive diagnosis in suspected cases, leading to an earlier treatment and enrollment in clinical trials. The extensive application of modern MR imaging-based techniques to the study of ALS has undoubtedly improved our understanding of disease pathophysiology and may have a role in the identification of potential biomarkers of disease progression. $\mathrm{AMRI}$ investigations of the motor and cognitive networks in patients with ALS have demonstrated an altered recruitment of regions normally devoted to the performance of a given task and/or the recruitment of additional areas in comparison with healthy subjects.

Nevertheless, there are many remaining challenges. New techniques need to be harnessed and integrated into clinical research and practice. New acquisition schemes and analysis procedures require standardization and optimization so that they can be used in multiple settings, both in natural history studies and treatment trials. From the data available, it is evident that combining different MR imaging modalities that are sensitive to different aspects of ALS pathology (eg, motor and extramotor) is a promising way to further increase our understanding of the mechanisms accounting for the accumulation of irreversible disability in this condition. Moreover, the emerging scenario from genetic studies indicates a consistent overlap between the pathogenetic changes in most forms of sporadic and familial ALS, ${ }^{108-110}$ thus suggesting that the application of neuroimaging in familial patients with ALS may allow identification of abnormalities that can hopefully be translated to the assessment of sporadic ALS.

\section{Acknowledgments}

The design and preparation of this review were performed under the auspices of the Gruppo di Studio "Neuroimmagini," Società Italiana di Neurologia. 


\section{References}

1. Ravits JM, La Spada AR. ALS motor phenotype heterogeneity, focality, and spread: deconstructing motor neuron degeneration. Neurology 2009;73: 805-11

2. Dion PA, Daoud H, Rouleau GA. Genetics of motor neuron disorders: new insights into pathogenic mechanisms. Nat Rev Genet 2009;10:769-82

3. Neumann M, Sampathu DM, Kwong LK, et al. Ubiquitinated TDP-43 in frontotemporal lobar degeneration and amyotrophic lateral sclerosis. Science 2006;314:130-33

4. Leigh PN, Abrahams S, Al-Chalabi A, et al. The management of motor neurone disease. J Neurol Neurosurg Psychiatry 2003;74(suppl 4):iv32-iv47

5. Andersen PM, Borasio GD, Dengler R, et al. EFNS task force on management of amyotrophic lateral sclerosis: guidelines for diagnosing and clinical care of patients and relatives. Eur J Neurol 2005;12:921-38

6. Chio A, Cucatto A, Calvo A, et al. Amyotrophic lateral sclerosis among the migrant population to Piemonte, northwestern Italy. J Neurol 1999;246: $175-80$

7. Millul A, Beghi E, Logroscino G, et al. Survival of patients with amyotrophic lateral sclerosis in a population-based registry. Neuroepidemiology 2005;25:114-19

8. Zoccolella S, Beghi E, Palagano G, et al. Predictors of delay in the diagnosis and clinical trial entry of amyotrophic lateral sclerosis patients: a population-based study. J Neurol Sci 2006;250:45-49

9. Brooks BR. Managing amyotrophic lateral sclerosis: slowing disease progression and improving patient quality of life. Ann Neurol 2009;65(suppl 1):S17-23

10. Brooks BR, Miller RG, Swash M, et al. El Escorial revisited: revised criteria for the diagnosis of amyotrophic lateral sclerosis. Amyotroph Lateral Scler Other Motor Neuron Disord 2000;1:293-99

11. Turner MR, Kiernan MC, Leigh PN, et al. Biomarkers in amyotrophic lateral sclerosis. Lancet Neurol 2009;8:94-109

12. Kato S, Shaw P, Wood-Allum C, et al. Amyotrophic lateral sclerosis. In: Dickson DW, ed. Neurodegeneration: The Molecular Pathology of Dementia and Movement Disorders. Basel, Switzerland: ISN Neuropath Press; 2003:350-71

13. Lule D, Ludolph AC, Kassubek JM. I-based functional neuroimaging in ALS: an update. Amyotroph Lateral Scler 2009;10:258-68

14. Goodin DS, Rowley HA, Olney RK. Magnetic resonance imaging in amyotrophic lateral sclerosis. Ann Neurol 1988;23:418-20

15. Abe K, Fujimura H, Kobayashi $Y$, et al. Degeneration of the pyramidal tracts in patients with amyotrophic lateral sclerosis: a premortem and postmortem magnetic resonance imaging study. J Neuroimaging 1997;7:208-12

16. Cheung G, Gawel MJ, Cooper PW, et al. Amyotrophic lateral sclerosis: correlation of clinical and MR imaging findings. Radiology 1995;194:263-70

17. Hecht MJ, Fellner F, Fellner C, et al. MRI-FLAIR images of the head show corticospinal tract alterations in ALS patients more frequently than T2-, T1and proton-density-weighted images. J Neurol Sci 2001;186:37-44

18. Hecht MJ, Fellner F, Fellner C, et al. Hyperintense and hypointense MRI signals of the precentral gyrus and corticospinal tract in ALS: a follow-up examination including FLAIR images. J Neurol Sci 2002;199:59-65

19. Ishikawa K, Nagura H, Yokota T, et al. Signal loss in the motor cortex on magnetic resonance images in amyotrophic lateral sclerosis. Ann Neurol 1993;33:218-22

20. Mirowitz S, Sartor K, Gado M, et al. Focal signal-intensity variations in the posterior internal capsule: normal MR findings and distinction from pathologic findings. Radiology 1989;172:535-39

21. Thorpe JW, Moseley IF, Hawkes CH, et al. Brain and spinal cord MRI in motor neuron disease. J Neurol Neurosurg Psychiatry 1996;61:314-17

22. Waragai M. MRI and clinical features in amyotrophic lateral sclerosis. Neuroradiology 1997;39:847-51

23. Basak M, Erturk M, Oflazoglu B, et al. Magnetic resonance imaging in amyotrophic lateral sclerosis. Acta Neurol Scand 2002;105:395-99

24. Charil A, Corbo M, Filippi M, et al. Structural and metabolic changes in the brain of patients with upper motor neuron disorders: a multiparametric MRI study. Amyotroph Lateral Scler 2009;10:269-79

25. Satoh JI, Tokumoto H, Kurohara K, et al. Adult-onset Krabbe disease with homozygous T1853C mutation in the galactocerebrosidase gene: unusual MRI findings of corticospinal tract demyelination. Neurology 1997;49: 1392-99

26. Kassubek J, Bretschneider V, Sperfeld AD. Corticospinal tract MRI hyperintensity in X-linked Charcot-Marie-Tooth Disease. J Clin Neurosci 2005; $12: 588-89$

27. Teriitehau C, Adamsbaum C, Merzoug V, et al. Subtle brain abnormalities in adrenomyeloneuropathy [in French]. J Radiol 2007;88(7-8 pt 1):957-61

28. Oba H, Araki T, Ohtomo K, et al. Amyotrophic lateral sclerosis: T2 shortening in motor cortex at MR imaging. Radiology 1993;189:843-46

29. Matsusue E, Sugihara S, Fujii S, et al. Cerebral cortical and white matter lesions in amyotrophic lateral sclerosis with dementia: correlation with MR and pathologic examinations. AJNR Am J Neuroradiol 2007;28:1505-10

30. Mori H, Yagishita A, Takeda T, et al. Symmetric temporal abnormalities on
MR imaging in amyotrophic lateral sclerosis with dementia. AJNR Am J Neuroradiol 2007;28:1511-16

31. Kassubek J, Unrath A, Huppertz HJ, et al. Global brain atrophy and corticospinal tract alterations in ALS, as investigated by voxel-based morphometry of 3-D MRI. Amyotroph Lateral Scler Other Motor Neuron Disord 2005;6:213-20

32. Mezzapesa DM, Ceccarelli A, Dicuonzo F, et al. Whole-brain and regional brain atrophy in amyotrophic lateral sclerosis. AJNR Am J Neuroradiol 2007;28:255-59

33. Tartaglia MC, Laluz V, Rowe A, et al. Brain atrophy in primary lateral sclerosis. Neurology 2009;72:1236-41

34. Terao S, Sobue G, Yasuda T, et al. Magnetic resonance imaging of the corticospinal tracts in amyotrophic lateral sclerosis. J Neurol Sci 1995;133:66-72

35. Mackenzie IR, Feldman HH. Ubiquitin immunohistochemistry suggests classic motor neuron disease, motor neuron disease with dementia, and frontotemporal dementia of the motor neuron disease type represent a clinicopathologic spectrum. J Neuropathol Exp Neurol 2005;64:730-39

36. Agosta F, Pagani E, Rocca MA, et al. Voxel-based morphometry study of brain volumetry and diffusivity in amyotrophic lateral sclerosis patients with mild disability. Hum Brain Mapp 2007;28:1430-38

37. Grosskreutz J, Kaufmann J, Fradrich J, et al. Widespread sensorimotor and frontal cortical atrophy in amyotrophic lateral sclerosis. BMC Neurol 2006 6:17

38. Chang JL, Lomen-Hoerth C, Murphy J, et al. A voxel-based morphometry study of patterns of brain atrophy in ALS and ALS/FTLD. Neurology 2005 65:75-80

39. Kato S, Hayashi H, Yagishita A. Involvement of the frontotemporal lobe and limbic system in amyotrophic lateral sclerosis: as assessed by serial computed tomography and magnetic resonance imaging. J Neurol Sci 1993;116:52-58

40. Grossman M, Anderson C, Khan A, et al. Impaired action knowledge in amyotrophic lateral sclerosis. Neurology 2008;71:1396-401

41. Thivard L, Pradat PF, Lehericy S, et al. Diffusion tensor imaging and voxe based morphometry study in amyotrophic lateral sclerosis: relationships with motor disability. J Neurol Neurosurg Psychiatry 2007;78:889-92

42. Roccatagliata L, Bonzano L, Mancardi G, et al. Detection of motor cortex thinning and corticospinal tract involvement by quantitative MRI in amyotrophic lateral sclerosis. Amyotroph Lateral Scler 2009;10:47-52

43. Kiernan JA, Hudson AJ. Frontal lobe atrophy in motor neuron diseases. Brain $1994 ; 117$ (pt 4):747-57

44. Ellis CM, Suckling J, Amaro E Jr, et al. Volumetric analysis reveals corticospinal tract degeneration and extramotor involvement in ALS. Neurology 2001;57:1571-78

45. Abrahams S, Goldstein LH, Suckling J, et al. Frontotemporal white matter changes in amyotrophic lateral sclerosis. J Neurol 2005;252:321-31

46. Valsasina P, Agosta F, Benedetti B, et al. Diffusion anisotropy of the cervical cord is strictly associated with disability in amyotrophic lateral sclerosis. J Neurol Neurosurg Psychiatry 2007;78:480-84

47. Agosta F, Rocca MA, Valsasina P, et al. A longitudinal diffusion tensor MRI study of the cervical cord and brain in amyotrophic lateral sclerosis patients. J Neurol Neurosurg Psychiatry 2009;80:53-55

48. Gredal O, Rosenbaum S, Topp S, et al. Quantification of brain metabolites in amyotrophic lateral sclerosis by localized proton magnetic resonance spectroscopy. Neurology 1997;48:878-81

49. Sarchielli P, Pelliccioli GP, Tarducci R, et al. Magnetic resonance imaging and 1H-magnetic resonance spectroscopy in amyotrophic lateral sclerosis. Neuroradiology 2001;43:189-97

50. Mitsumoto H, Ulug AM, Pullman SL, et al. Quantitative objective markers for upper and lower motor neuron dysfunction in ALS. Neurology 2007;68: 1402-10

51. Block W, Karitzky J, Traber F, et al. Proton magnetic resonance spectroscopy of the primary motor cortex in patients with motor neuron disease: subgroup analysis and follow-up measurements. Arch Neurol 1998;55:931-36

52. Jones AP, Gunawardena WJ, Coutinho CM, et al. Preliminary results of proton magnetic resonance spectroscopy in motor neurone disease (amyotrophic lateral sclerosis). J Neurol Sci 1995;129(suppl):85-89

53. Pioro EP, Antel JP, Cashman NR, et al. Detection of cortical neuron loss in motor neuron disease by proton magnetic resonance spectroscopic imaging in vivo. Neurology 1994;44:1933-38

54. Rooney WD, Miller RG, Gelinas D, et al. Decreased N-acetylaspartate in motor cortex and corticospinal tract in ALS. Neurology 1998;50:1800-05

55. Pohl C, Block W, Karitzky J, et al. Proton magnetic resonance spectroscopy of the motor cortex in 70 patients with amyotrophic lateral sclerosis. $\mathrm{Arch} \mathrm{Neu}$ rol 2001;58:729-35

56. Kalra S, Arnold DL. Magnetic resonance spectroscopy for monitoring neuronal integrity in amyotrophic lateral sclerosis. Adv Exp Med Biol 2006;576: 275-82, discussion 361-63

57. Kalra S, Hanstock CC, Martin WR, et al. Detection of cerebral degeneration in amyotrophic lateral sclerosis using high-field magnetic resonance spectroscopy. Arch Neurol 2006;63:1144-48

58. Nelles M, Block W, Traber F, et al. Combined 3T diffusion tensor tractogra- 
phy and 1H-MR spectroscopy in motor neuron disease. AJNR Am J Neuroradiol 2008;29:1708-14

59. Wang S, Poptani H, Woo JH, et al. Amyotrophic lateral sclerosis: diffusiontensor and chemical shift MR imaging at 3.0 T. Radiology 2006;239:831-38

60. Pyra T, Hui B, Hanstock C, et al. Combined structural and neurochemical evaluation of the corticospinal tract in amyotrophic lateral sclerosis. Amyotroph Lateral Scler 2010;11:157-65

61. Cwik VA, Hanstock CC, Allen PS, et al. Estimation of brainstem neuronal loss in amyotrophic lateral sclerosis with in vivo proton magnetic resonance spectroscopy. Neurology 1998;50:72-77

62. Ellis CM, Simmons A, Andrews C, et al. A proton magnetic resonance spectroscopic study in ALS: correlation with clinical findings. Neurology 1998;51: 1104-09

63. Abe K, Takanashi M, Watanabe $\mathrm{Y}$, et al. Decrease in $\mathrm{N}$-acetylaspartate/creatine ratio in the motor area and the frontal lobe in amyotrophic lateral sclerosis. Neuroradiology 2001;43:537-41

64. Kaufmann P, Pullman SL, Shungu DC, et al. Objective tests for upper motor neuron involvement in amyotrophic lateral sclerosis (ALS). Neurology 2004;62:1753-57

65. Bowen BC, Pattany PM, Bradley WG, et al. MR imaging and localized proton spectroscopy of the precentral gyrus in amyotrophic lateral sclerosis. AJNR Am J Neuroradiol 2000;21:647-58

66. Cosottini M, Giannelli M, Siciliano G, et al. Diffusion-tensor MR imaging of corticospinal tract in amyotrophic lateral sclerosis and progressive muscular atrophy. Radiology 2005;237:258-64

67. Ellis CM, Simmons A, Jones DK, et al. Diffusion tensor MRI assesses corticospinal tract damage in ALS. Neurology 1999;53:1051-58

68. Toosy AT, Werring DJ, Orrell RW, et al. Diffusion tensor imaging detects corticospinal tract involvement at multiple levels in amyotrophic lateral sclerosis. J Neurol Neurosurg Psychiatry 2003;74:1250-57

69. Abe O, Yamada H, Masutani Y, et al. Amyotrophic lateral sclerosis: diffusion tensor tractography and voxel-based analysis. NMR Biomed 2004;17:411-16

70. Iwata NK, Aoki S, Okabe S, et al. Evaluation of corticospinal tracts in ALS with diffusion tensor MRI and brainstem stimulation. Neurology 2008;70:528-32

71. Graham JM, Papadakis N, Evans J, et al. Diffusion tensor imaging for the assessment of upper motor neuron integrity in ALS. Neurology 2004;63:2111-19

72. Schimrigk SK, Bellenberg B, Schluter M, et al. Diffusion tensor imaging-based fractional anisotropy quantification in the corticospinal tract of patients with amyotrophic lateral sclerosis using a probabilistic mixture model. AJNR Am J Neuroradiol 2007;28:724-30

73. Senda J, Ito M, Watanabe $\mathrm{H}$, et al. Correlation between pyramidal tract degeneration and widespread white matter involvement in amyotrophic lateral sclerosis: a study with tractography and diffusion-tensor imaging. Amyotroph Lateral Scler 2009;10:288-94

74. Ciccarelli O, Behrens TE, Altmann DR, et al. Probabilistic diffusion tractography: a potential tool to assess the rate of disease progression in amyotrophic lateral sclerosis. Brain 2006;129:1859-71

75. Sage CA, Peeters RR, Gorner A, et al. Quantitative diffusion tensor imaging in amyotrophic lateral sclerosis. Neuroimage 2007;34:486-99

76. Hughes JT. Pathology of amyotrophic lateral sclerosis. Adv Neurol 1982; 36:61-74

77. Lawyer T Jr, Netsky MG. Amyotrophic lateral sclerosis. AMA Arch Neurol Psychiatry 1953;69:171-92

78. da Rocha AJ, Oliveira AS, Fonseca RB, et al. Detection of corticospinal tract compromise in amyotrophic lateral sclerosis with brain MR imaging: relevance of the T1-weighted spin-echo magnetization transfer contrast sequence. AJNR Am J Neuroradiol 2004;25:1509-15

79. Tanabe JL, Vermathen M, Miller R, et al. Reduced MTR in the corticospinal tract and normal T2 in amyotrophic lateral sclerosis. Magn Reson Imaging 1998;16:1163-69

80. Kato Y, Matsumura K, Kinosada Y, et al. Detection of pyramidal tract lesions in amyotrophic lateral sclerosis with magnetization-transfer measurements. AJNR Am J Neuroradiol 1997;18:1541-47

81. Filippi M, Rocca MA. Magnetization transfer magnetic resonance imaging of the brain, spinal cord, and optic nerve. Neurotherapeutics 2007;4:401-13

82. Konrad C, Henningsen $\mathrm{H}$, Bremer J, et al. Pattern of cortical reorganization in amyotrophic lateral sclerosis: a functional magnetic resonance imaging study. Exp Brain Res 2002;143:51-56

83. Konrad C, Jansen A, Henningsen H, et al. Subcortical reorganization in amyotrophic lateral sclerosis. Exp Brain Res 2006;172:361-69
84. Schoenfeld MA, Tempelmann C, Gaul C, et al. Functional motor compensation in amyotrophic lateral sclerosis. J Neurol 2005;252:944-52

85. Stanton BR, Williams VC, Leigh PN, et al. Altered cortical activation during a motor task in ALS: evidence for involvement of central pathways. J Neurol 2007;254:1260-67

86. Tessitore A, Esposito F, Monsurro MR, et al. Subcortical motor plasticity in patients with sporadic ALS: an fMRI study. Brain Res Bull 2006;69:489-94

87. Neary D, Snowden JS, Gustafson L, et al. Frontotemporal lobar degeneration: a consensus on clinical diagnostic criteria. Neurology 1998;51:1546-54

88. Murphy JM, Henry RG, Langmore S, et al. Continuum of frontal lobe impairment in amyotrophic lateral sclerosis. Arch Neurol 2007;64:530-34

89. Avants B, Khan A, McCluskey L, et al. Longitudinal cortical atrophy in amyotrophic lateral sclerosis with frontotemporal dementia. Arch Neurol 2009; 66:138-39

90. Agosta F, Gorno-Tempini ML, Pagani E, et al. Longitudinal assessment of grey matter contraction in amyotrophic lateral sclerosis: a tensor based morphometry study. Amyotroph Lateral Scler 2009;10:168-74

91. Ciccarelli O, Behrens TE, Johansen-Berg $\mathrm{H}$, et al. Investigation of white matter pathology in ALS and PLS using tract-based spatial statistics. Hum Brain Mapp 2009;30:615-24

92. Sach M, Winkler G, Glauche V, et al. Diffusion tensor MRI of early upper motor neuron involvement in amyotrophic lateral sclerosis. Brain 2004; $127: 340-50$

93. Sage CA, Van Hecke W, Peeters R, et al. Quantitative diffusion tensor imaging in amyotrophic lateral sclerosis: revisited. Hum Brain Mapp 2009;30:3657-75

94. Abrahams S, Goldstein LH, Simmons A, et al. Word retrieval in amyotrophic lateral sclerosis: a functional magnetic resonance imaging study. Brain 2004;127:1507-17

95. Lule D, Diekmann V, Anders S, et al. Brain responses to emotional stimuli in patients with amyotrophic lateral sclerosis (ALS). J Neurol 2007;254:519-27

96. Wijesekera LC, Leigh PN. Amyotrophic lateral sclerosis. Orphanet J Rare Dis 2009;4:3

97. Suhy J, Miller RG, Rule R, et al. Early detection and longitudinal changes in amyotrophic lateral sclerosis by (1)H MR spectroscopy. Neurology 2002; 58:773-79

98. Unrath A, Ludolph AC, Kassubek J. Brain metabolites in definite amyotrophic lateral sclerosis: a longitudinal proton magnetic resonance spectroscopy study. J Neurol 2007;254:1099-106

99. Rule RR, Suhy J, Schuff N, et al. Reduced NAA in motor and non-motor brain regions in amyotrophic lateral sclerosis: a cross-sectional and longitudinal study. Amyotroph Lateral Scler Other Motor Neuron Disord 2004;5:141-49

100. Blain CR, Williams VC, Johnston C, et al. A longitudinal study of diffusion tensor MRI in ALS. Amyotroph Lateral Scler 2007;8:348-55

101. Kalra S, Cashman NR, Genge A, et al. Recovery of N-acetylaspartate in corticomotor neurons of patients with ALS after riluzole therapy. Neuroreport 1998;9:1757-61

102. Kalra S, Tai P, Genge A, et al. Rapid improvement in cortical neuronal integrity in amyotrophic lateral sclerosis detected by proton magnetic resonance spectroscopic imaging. J Neurol 2006;253:1060-63

103. Kalra S, Cashman NR, Caramanos Z, et al. Gabapentin therapy for amyotrophic lateral sclerosis: lack of improvement in neuronal integrity shown by MR spectroscopy. AJNR Am J Neuroradiol 2003;24:476-80

104. Kalra S, Genge A, Arnold DL. A prospective, randomized, placebo-controlled evaluation of corticoneuronal response to intrathecal BDNF therapy in ALS using magnetic resonance spectroscopy: feasibility and results. Amyotroph Lateral Scler Other Motor Neuron Disord 2003;4:22-26

105. Schenck JF, Zimmerman EA. High-field magnetic resonance imaging of brain iron: birth of a biomarker? NMR Biomed 2004;17:433-45

106. Lule D, Diekmann V, Kassubek J, et al. Cortical plasticity in amyotrophic lateral sclerosis: motor imagery and function. Neurorehabil Neural Repair 2007;21:518-26

107. Mohammadi B, Kollewe K, Samii A, et al. Changes of resting state brain networks in amyotrophic lateral sclerosis. Exp Neurol 2009;217:147-53

108. Corrado L, Del Bo R, Castellotti B, et al. Mutations of FUS gene in sporadic amyotrophic lateral sclerosis. J Med Genet 2010;47:190-94

109. Belzil VV, Valdmanis PN, Dion PA, et al. Mutations in FUS cause FALS and SALS in French and French Canadian populations. Neurology 2009;73:117679. Epub 2009 Sep 9

110. Kirby J, Goodall EF, Smith W, et al. Broad clinical phenotypes associated with TAR-DNA binding protein (TARDBP) mutations in amyotrophic latera sclerosis. Neurogenetics 2010;11:217-25 